\title{
2011 Reviewers
}

Rema Adel Afifi

Siddharth Agarwal

Silvia Amesty

Tony Antoniou

Yumiko Aratani

Sonya Arreola

Joel Ashby

Dankmar Böhning

Michael D. M. Bader

Josh Bamberger

Briana Barocas

Gregorio Barrio

Charles E. Begley

Peter Beilenson

Leo Beletsky

George Bigelow

Elena Bilotta

Ingrid A. Binswanger

Jennifer L. Black

Keosha Bond

Lee Bone

Carla Boutin-Foster

Anthony Braga

Randall Brown

Larry Burd

Jessica Burke

Waleska Caiaffa

Joanne Cannavo

Carolyn C. Cannuscio

David Celentano

Magdalena Cerda

Chuan-Yu Chen

Jason Corburn

Nicholas Corsaro

Natalie Crawford

Evelyn P. Davila

Marlou de Kroon

Andrea De Silva - Sanigorski

Don DesJarlais

Theresa Diaz

Douglas Dieterich

Charles DiMaggio

Jonathan Ellen

Christopher G. Ellison

Alex Ezeh
Stuart Feldman

Sally Findley

Shawn Fraser

Maysoun Freij

Sharon Friel

Robert Fullilove

Mindy Fullilove

Francesca Gany

Richard S Garfein

Lillian Gelberg

Danielle German

Robyn Gershon

Christian T. Gloria

Phillip Graham

Theresa Grant

Carolyn Greene

Michael Gusmano

V. Anna Gyarmathy

Simon Hödl

Jack Hadley

Judy Hahn

Ted Hammett

Melvin Hampton

Helena Hansen

Trudy Harpham

Robert Heimer

Margaret Hellard

Lori Hensic

Don Hoover

Stephen W Hwang

Simeon Isezuo

Paul Jargowsky

Jacky M Jennings

David Jernigan

Camara Jones

Mitchell Katz

Gabe Kelen

Brian C Kelly

Jacqueline Kerr

Deanna Kerrigan

Daniel Kim

Amy Knowlton

Beryl Koblin

Cynthia Kratzke

Jacob Kumaresan
Yadlapalli Kusuma

Naa Oyo A. Kwate

Stephen Lankenau

Kristian Larsen

Carl Latkin

Kathryn Lee

LaVonna Blair Lewis

Peter Lin

James Angelo Listorti

Gina Schellenbaum Lovasi

Greg Lucas

Melanie Lutenbacher

Cynthia Lyles

Nyovani JAnet Madise

Megan Ruth Mahoney

Ruth E. Malone

Alex D. Manini

Brandon D. L. Marshall

Stephen Matthews

Geraldine McQuillan

Shruti Mehta

Lynne Messer

Meredith Minkler

Mark Randolph

Montgomery

Julia Morinis

Peter Muennig

Mahasin Mujahid

Paul Muntner

Arijit Nandi

Denis Nash

Amy Nunn

Amani M. Nuru-Jeter

Ann Oleary

Bolajoko O. Olusanya

Danielle C. Ompad

Benjamin Ortiz

David Osrin

David Ostrow

Ana Bertha Pérez- Lizaur

Susan Palsbo

Jenna Panter

Gregory Pappas

Richard Parker

Diana Parra 
Preeti Pathela

Ajay Bhaskar Pawar

Lucy Peipins

D’Ann Penner

Penelope Phillips-Howard

Robin A. Pollini

Dwayne Porter

Carmen Portillo

Roslyn Poulos

Elizabeth Racine

Elizabeth Reed

Josiah Rich

Thomas C. Ricketts

Elise D. Riley

Susan Robinson

Paul Robinson

Dori Rosenberg

Andrew Rundle

Brian Saelens

Monika Safford
Jonathan Samet

William A. Satariano

David Savitz

Peter Schantz

John Schneider

Eric W Schrimshaw

Steven Schroeder

Jenny Scott

Jan Semenza

Ketan Shankardass

Donna Shelley

Steve Shoptaw

John Song

Anne Spaulding

Ron Stall

Thomas John Stopka

Carla Storr

Shiela M. Strauss

Andrew H. Talal

Karin Tobin
Mark Tyndall

Marcelo Luis Urquia

Tom Valente

Delfien Van Dyck

Lisa Vinikoor-Imler

Stephanie Wahab

Renee E. Walker

Deborah Wallace

Margaret Wallhagen

Anne Wallis

Catherine Waters

Daniel Webster

Linda Weiss

Douglas Wiebe

Sara Wilcox

Richard Wolitski

Irene Yen

Deborah Zador

Shannon Zenk

Jeanette Y. Ziegenfuss 